\title{
Serum proteomic profiling at diagnosis predicts clinical course, and need for intensification of treatment in inflammatory bowel disease.
}

Kalla $\mathrm{R}^{1,2^{*}}$, Adams $\mathrm{AT}^{1,3^{*}}$, Bergemalm $\mathrm{D}^{4}$, Vatn $\mathrm{S}^{5}$, Kennedy $\mathrm{NA}^{1,6}$, Ricanek $\mathrm{P}^{5,15}$, Lindstrom $\mathbf{J}^{7,15}$, Ocklind $\mathrm{A}^{8}$, Hjelm $\mathrm{F}^{8}$, Ventham $\mathrm{NT}^{1}$, Ho $\mathrm{GT}^{2}$, Petren $\mathrm{C}^{8}$, IBD-Character Consortium, Repsilber $\mathrm{D}^{9}$, Söderholm $\mathrm{J}^{10}$, Pierik $\mathrm{M}^{11}$, D’Amato $\mathrm{M}^{12,13}$, Gomollón $\mathrm{F}^{14}$, Olbjorn $\mathrm{C}^{5,15}$, Jahnsen $\mathrm{J}^{5,15}$, Vatn $\mathrm{MH}^{15}$, Halfvarson $\mathrm{J}^{4}$, Satsangi $\mathrm{J}^{1,3}$.

${ }^{1}$ Institute of Genetics and Molecular Medicine, University of Edinburgh, United Kingdom

${ }^{2}$ MRC Centre for Inflammation Research, Queens Medical Research Institute, University of Edinburgh, United Kingdom

${ }^{3}$ Translational Gastroenterology Unit, Nuffield Department of Medicine, Experimental Medicine Division, University of Oxford, John Radcliffe Hospital, Oxford, United Kingdom

${ }^{4}$ Department of Gastroenterology, Faculty of Medicine and Health, Örebro University, SE 70182 Örebro, Sweden

${ }^{5}$ Department of Gastroenterology, Akershus University Hospital, Lørenskog, Norway

${ }^{6}$ Exeter IBD and Pharmacogenetics group, University of Exeter, United Kingdom

${ }^{7}$ Health Services Research Unit, Akershus University Hospital, Lørenskog, Norway

${ }^{8}$ Olink Proteomics, Uppsala, Sweden

${ }^{9}$ School of Medical Sciences, Örebro University, Örebro, Sweden

(C) Crown copyright 2020.

This article contains public sector information licensed under the Open Government Licence v3.0 (http://www.nationalarchives.gov.uk/doc/open-government-licence/version/3/). 
${ }^{10}$ Department of Surgery and Clinical and Experimental Medicine, Linköping University, Linköping, Sweden

${ }^{11}$ Maastricht University Medical Centre (MUMC), Department of Gastroenterology and Hepatology, Maastricht, Netherlands

${ }^{12}$ Biocruces Health Research Institute, Molecular Genetics of Digestive Diseases, Cruces, Bilbao, Spain.

${ }^{13}$ School of Biological Sciences, Monash University, Victoria, Australia

${ }^{14}$ HCU “Lozano Blesa,” IIS Aragón, Zaragoza, Spain

${ }^{15}$ Institute of Clinical Medicine, Campus Ahus, University of Oslo, Oslo, Norway.

Corresponding Professor J Satsangi, Dr Rahul Kalla, Dr Alex Adams. 


\section{Summary}

Background: Success in personalised medicine in complex disease is critically dependent on biomarker discovery. We profiled serum proteins using a novel proximity extension assay (PEA) to identify diagnostic and prognostic biomarkers in inflammatory bowel disease (IBD).

Methods: We conducted a prospective case-control study in an inception cohort of 552 patients (328 IBD, 224 non-IBD), profiling proteins recruited across 6 centres. Treatment escalation was characterised by the need for biological agents or surgery after initial disease remission. Nested leave-one-out cross validation was used to examine the performance of diagnostic and prognostic proteins.

Results: A total of 66 serum proteins differentiated IBD from symptomatic non-IBD controls including MMP-12 (Holm adjusted $\left.\mathrm{p}=4.1 \times 10^{-23}\right)$ and OSM $\left(\mathrm{p}=3.7 \times 10^{-16}\right)$. Nine of these proteins associate with cis- germline variation (59 independent SNPs). Fifteen proteins, all members of TNF independent pathways including IL-1 and OSM predicted escalation, over a median follow-up of 518 (IQR 224-756) days. Nested cross-validation of the entire data set allows characterisation of 5-protein-models (96\% comprising five core proteins ITGAV, EpCAM, IL18, SLAMF7, and IL8) which define a high-risk subgroup in IBD (HR 3.90, CI: 2.43-6.26), or allows distinct 2, and 3 protein models for UC and CD respectively.

Conclusion: We have characterised a simple oligo-protein panel that has the potential to identify IBD from symptomatic controls and to predict future disease course. Further prospective work is required to validate our findings.

Keywords: Crohn's disease, proteins, genetics, inflammatory bowel diseases (IBD), ulcerative colitis, OSM, prognosis, outcomes, protein quantitative trait loci, proximity extension assay, 


\section{Introduction}

Personalised medicine is now a major priority in healthcare research. Programmes such as the $7^{\text {th }}$ framework programme for research and technological development and 100,000 genomes project (www.genomicsengland.co.uk) in the UK prioritise the discovery and validation of novel biomarkers in human diseases ${ }^{1}$. This impetus to redefine clinical practice coupled with an expanding therapeutic choice of biological agents, and small molecules has driven interest in risk-stratifying patients at diagnosis in inflammatory bowel disease (IBD) $)^{2-4}$.

There have been recent scientific advances catalysing biomarker discovery studies. It is now apparent that genes that contribute to prognosis in Crohn's disease (CD) are distinct from those that predict disease susceptibility ${ }^{4}$. Studies in both adults and children have demonstrated that patients with a progressive disease display a unique transcriptional signature ${ }^{3,5-7}$. Critically for translation, emergent data demonstrate that early biomarkerdriven therapeutic interventions can improve disease outcomes in $\mathrm{CD}^{8}$.

Despite significant progress in multi-omic biomarker discoveries, none have been translated into routine clinical practice. Markers such as C-reactive protein (CRP) have shown clinical utility in disease susceptibility, activity and behaviour ${ }^{1}$. Faecal calprotectin (FC) however has emerged to date as the most reliable and accurate diagnostic protein biomarker in IBD $^{9}$.

Recently, randomised trial data demonstrate that early biomarker-driven therapeutic interventions based on FC can improve disease outcomes in $\mathrm{CD}^{8}$. However, there are welldescribed limitations of faecal testing in clinical care ${ }^{2,10,11}$ highlighting the need for bloodbased markers to maximise uptake and acceptability. 
Multiprotein signatures have potentially diverse clinical applications from early detection of IBD to disease classification and behaviour, response to therapy, and monitoring disease activity. Technological limitations in multi-protein profiling have recently been overcome $^{12,13}$, with the discovery of innovative approaches for multiplexing biological samples utilizing minimal sample volume but providing a highly sensitive and specific immunoassay. Proximity extension assays (PEA) are antibody-based methods that utilise two or more DNA-tagged aptamers or antibodies that bind when in close proximity to the target protein or protein complex. PEA allows multiplexing with 1 microlitre $(\mu \mathrm{L})$ sample consumption, and a high sensitivity and specificity for proteins of interest ${ }^{12,13}$.

In this report, we explore the diagnostic and prognostic performance of circulating PEA based proteins markers in IBD and their association with germline variations. Our study demonstrates that protein panels can predict disease and its course.

\section{Materials and Methods}

\section{Study Design}

We conducted a prospective, multi-centre case-control study in patients with suspected or confirmed IBD, recruited at presentation as out-patients or as in-patients across 6 clinical centres in Europe (EU Character reference no. 305676) from May 2012 until September 2015. The diagnosis of ulcerative colitis (UC), CD and IBD-unclassified (IBDU) was based on internationally accepted criteria, following thorough clinical, microbiological, endoscopic, histological, and radiological evaluation. The control group consisted of patients with gastrointestinal symptoms (symptomatic controls), who had no discernible evidence of IBD at any time during follow-up. We recorded information on demographics, clinical characteristics according to the Montreal and Paris classification and details of drug therapies at baseline, i.e. at recruitment (Table 1) ${ }^{14-16}$. Treatment naivety within the IBD cohort was 
defined as no exposure to any IBD related medical therapies such as steroids, 5-

aminosalicylic acid (5-ASA), immunomodulators and biologics (Supplementary Table 1).

Blood samples for protein profiles and genotyping and stool samples for faecal calprotectin were collected at baseline, i.e. at the time of recruitment. High sensitivity CRP (hsCRP), albumin, and faecal calprotectin were re-assayed in a single batch at the end of recruitment. Patients with IBD were followed prospectively and information on clinical outcomes were collected during follow-up. Treatment escalation was defined as the need for a biologic, ciclosporin or surgery, instituted for disease flare after initial induction therapy and aiming to induce disease remission. In UC, the definition of treatment escalation also included colectomy during index admission.

All centres were granted local ethics approval for this study and all patients gave written informed consent prior to inclusion.

\section{Sample collection and processing}

We collected blood samples (Vacuette ${ }^{\circledR}$ gel tube with clot activator) and separated serum after centrifugation at $2,000 \mathrm{G}$ for ten minutes and within two hours of sampling. All serum were subsequently stored as aliquots at $-80^{\circ} \mathrm{C}$ until further use. Whole-blood leukocyte DNA was extracted using the Nucleon BACC 3 DNA extraction kit (GE healthcare, Buckinghamshire, UK). We genotyped patients using the Illumina OmniExpressExome-8 Bead Chip (Illumina, San Diego, CA, USA).

\section{Serum protein profiling}

We generated a candidate list of proteins based on the 163 IBD risk genes identified from genome-wide association studies ${ }^{17}$ and from the existing literature relating to the pathogenesis in IBD. After thorough quality control, assay analyses and validation, we built five unique multiplex protein panels, each comprising of 92 proteins. Thus, a total of 460 proteins were analysed. These proteins are involved in various IBD-related mechanisms, such 
as inflammation, immune regulation, metabolism and cell-cell signalling, and are listed in

\section{Supplementary Table 2.}

We used the Proximity Extension Assay (PEA) technology to measure protein concentrations 12,13. The methodology has been described in detail elsewhere ${ }^{13}$. Briefly, pairs of antibodies are used towards each target antigen. When both antibodies bind to the same antigen in close proximity, attached oligonucleotides hybridize. The oligonucleotide templates are extended and amplified using polymerase chain reaction (PCR) (96.96, Dynamic Array IFC, Fluidigm Biomark) on a Biomark HD Instrument. For each panel, 92 oligonucleotide-labelled antibody probe pairs are allowed to bind to their respective target present in the sample. All samples were processed at Olink Proteomics, Uppsala, Sweden.

To minimize inter- and intra-plate variation, raw data (qPCR Ct values) were normalized using internal controls in each multiplex reaction, negative controls and an inter-plate control on each plate, and then transformed using a pre-determined correction factor. The preprocessed data were reported as an arbitrary unit, i.e. normalized protein expression (NPX) on a $\log 2$ scale as described previously ${ }^{12,13,18}$. A high NPX represents high protein concentration and a low NPX represent low protein concentration. Limit of detection (LOD) for each protein probe was defined as the mean plus three standard deviations of the negative controls.

To reduce the effect of biologically irrelevant differences or non-informative protein features, we first excluded $147 / 460$ proteins where $>50 \%$ of samples were below the LOD, and then excluded 33 samples in which $>20 \%$ of the remaining proteins were below the LOD. After quality control, a total of 313 proteins were analysed in 552 patients. 


\section{Statistical analysis}

\section{Manuscript Doi: 10.1093/ecco-jcc/jjaa230}

We used R 3.4.4 (R Foundation for Statistical Computing, Vienna, Austria) and Julia 1.1.0 ${ }^{19}$ for analysis. Data were corrected for centre batch effects using ComBat. P-values were adjusted for multiple testing (Holm correction) ${ }^{20}$. Survival analysis was performed using univariate Cox proportional hazard models and including age and sex as covariates. Hazard ratios (HRs) are calculated from cox regression coefficients. HR represents the relative risk associated with a one unit increase in expression of the relevant protein. Diagnostic analysis including sub-analysis differentiating UC from CD was performed using binomial logistic regression. We constructed models and characterised their predictive performance using a rigorous nested cross-validation approach wherein feature selection and parameter estimation were performed in an inner leave-one-out (LOO) cross-validation loop, with the model performance assessed using the unseen outer LOO sample. Reported performance of the models is based on the combined performance in each outer LOO sample of the models derived in their respective inner loops. Models were constrained to include age and sex, with proteins added in a forward stepwise approach based on akaike information criterion (AIC). The number of included proteins was based on the AIC evidence ratio assessed in the first $10 \%$ of outer loops after which models were constrained to the selected number of proteins to reduce computation. No pre-selection or filtering of the proteins by any criteria was used prior to the cross-validation. Classification was based on the optimum threshold from ROC analysis of the outer cross-validation loop. Randomly permuted data $(n=50)$ were analysed with the same technique with true data outperforming every permuted dataset.

Genome Studio files were imported into R for sex mismatch removal, and further analysis. Protein quantitative trait loci (pQTLs) were found using the matrix eQTL package ${ }^{21}$ with a distance threshold of $300 \mathrm{~Kb}$ and a MAF threshold of $>0.1$. Age and sex were included as 
covariates, and Holm correction was applied to $\mathrm{p}$ values. Further sub-analysis was performed with treatment exposure, sex, age, BMI, clinical centre, and smoking status as covariates.

\section{Results}

\section{Differentially expressed protein markers in Inflammatory Bowel Diseases}

After quality control, a total of 313 proteins were analysed in 552 patients recruited across six IBD centres in Europe (Table 1). Linear models with age and sex as covariates identified a total of 66 protein markers that showed significant differential expression between IBD $(n=328)$ and controls ( $n=224$, Figure 1 and Supplementary Table 3), including Matrix Metallopeptidase-12 (MMP-12, $\log _{2}$ fold change $\left.\left(\log _{2} \mathrm{FC}\right)=0.87, \mathrm{p}=4.1 \times 10^{-23}\right)$ and Oncostatin-M $\left(\mathrm{OSM}, \log _{2} \mathrm{FC}=0.81, \mathrm{p}=3.7 \times 10^{-16}\right)$. Over-expression in IBD was more frequent at higher significance levels $(\mathrm{p}=0.01)$, with the top 12 proteins all being over-expressed. Of the proteins down-regulated in IBD, the most significant ones include Growth ArrestSpecific-6 (GAS6) and Integrin alpha-V (ITGAV).

There were 55 protein markers that were significantly differentially expressed in CD compared to controls (Supplementary Table 4); the most significant being CXCL9 $\left(\log _{2} \mathrm{FC}\right.$ $\left.=1.02, \mathrm{p}=5.0 \times 10^{-15}\right)$ and $\mathrm{OSM}\left(\log _{2} \mathrm{FC}=0.82, \mathrm{p}=5.8 \times 10^{-12}\right)$. In UC, 46 protein markers had significant expression differences compared to controls (Supplementary Table 5), including MMP-12 $\left(\log _{2} \mathrm{FC}=1.14, \mathrm{p}=3.6 \times 10^{-26}\right)$ and Granzyme-B $\left(\log _{2} \mathrm{FC}=1.54, \mathrm{p}=7.9 \times 10^{-23}\right)$. A total of 5 proteins showed significant expression differences between UC and CD (Supplementary Table 6, Figure 1B), all were significantly different between CD and controls, and differed further in the same direction in UC. A clinically useful model to distinguish between CD and UC could not be established, since the accuracy of the best performing classifier (consisting of age, sex, and expression of six proteins) was only $68.0 \%$. 


\section{Manuscript Doi: 10.1093/ecco-jcc/jjaa230}

Correlations between protein expression and inflammatory markers are shown in

\section{Supplementary Figure S1.}

\section{Diagnosis of IBD with PEAs and inflammatory markers}

We next examined the diagnostic performance of PEA-based protein models using the nested cross-validation approach, independent of the differential expression analysis, using all proteins profiled in this study. Fitting logistic regression models comprising age, sex, and 6 protein expression values in a nested cross-validation approach was 79.8\% (95\% confidence interval (CI): 76.4-83.2) accurate at distinguishing IBD from controls (sensitivity 83.1\%, CI: 79.1-87.2; specificity $74.8 \%$, CI: $69.0-80.5)$. The proteins selected by each inner crossvalidation loop were stable, comprising Granzyme-B (selected by $100 \%$ of inner loops), MMP12 (100\%), Gas6 (99.8\%), Interleukin-7 (IL7, 99.6\%), Interleukin-8 (IL8, 99.6\%), and Extracellular matrix metalloproteinase inducer (EMMPRIN, 99.3\%).

This model outperformed an hsCRP-model with age and sex, which had a sensitivity of 77.5\% (CI: 72.7-82.3), specificity of $27.8 \%$ (CI: $21.5-34.0)$ and an accuracy of $57.2 \%$ (CI: 52.9-61.7) (Supplementary Table 7). An FC model with age and sex performed better (sensitivity $85.4 \%$, CI: 78.1-92.7; specificity $88.4 \%$, CI: 78.8-98.0, accuracy $86.4 \%$, CI: 80.592.2\%), however FC suffers from poor uptake, with only $30.4 \%$ of patients having a result between 30 days prior- and 7 days post-inclusion.

The PEA-based models performed similarly in UC and CD (accuracy $78.4 \%$ and $77.7 \%$ respectively), and separate analysis of CD and UC did not produce more accurate models. FC was more sensitive in UC compared to CD (90.7, CI: 83.0-98.5 vs 77.4\%, CI: 62.7-92.1; $\chi^{2}$ $\mathrm{p}=1.2 \times 10^{-12}$ ), yielding an improved accuracy of $89.7 \%$, CI: $83.6-95.7$ vs $83.8 \%$, CI: 75.4-92.2 (Supplementary Table 7). 


\section{Individual proteins associated with treatment escalation}

In order to identify proteins that associate with treatment escalation, we analysed data from

279 patients with confirmed IBD from whom follow up data were available (Table 2 and

Supplementary Table 8). Patients who required escalation were younger (median age 28 vs

$33, \mathrm{p}=0.02)$, more likely to be male (58.2 vs $\left.51.4 \%, \chi^{2} \mathrm{p}>0.05\right)$, and have $\mathrm{CD}(58.2$ vs $34.4 \%$, $\left.\chi^{2} \mathrm{p}=0.004\right)$. There was no significant association between treatment escalation and smoking status amongst patients with CD or UC.

Cox models were created to identify protein markers individually associated with treatment escalation in IBD, accounting for age and sex. Fifteen proteins (Figure 2 and Table 3) were significantly associated with treatment escalation in IBD, including ITGAV $\left(p=3.2 \times 10^{-6}\right)$ and EpCAM $\left(p=1.7 \times 10^{-4}\right)$. Adjusting for treatment naivety did not influence the top differentially expressed proteins among patients with IBD. In UC ( $\mathrm{n}=143), 22$ proteins were significantly associated with treatment escalation (Supplementary Table 9), but in CD ( $\mathrm{n}=112)$ no individual proteins achieved significance, although the results were correlated with those obtained for UC alone $\left(\mathrm{r}=0.56, \mathrm{p}=6.6 \times 10^{-15}\right)$.

\section{Nested cross-validation stratifies disease sub-groups that associate with treatment escalation}

Models to define need for treatment escalation consisting of age, sex, IBD subtype, and PEAprotein expression values were generated in each inner leave-one-out cross-validation loop and tested in the outer loop. The models selected were highly stable. A series of 5-protein models had highest predictive accuracy, with $96 \%$ of these models consisting of the same 5 proteins (ITGAV, EpCAM, IL18, SLAMF7, and IL8).

These models defined by cross-validation had 80.0\% (CI: 75.3-84.7\%) accuracy (sensitivity 47.6\% (CI: 35.3-60.0\%), specificity 89.6\% (CI: 85.5-93.7), with a positive likelihood ratio $(\mathrm{LR}+) 4.59$ [CI: 2.86-7.36], and negative likelihood ratio(LR-) 0.58 [CI: 0.46-0.74]). The 
high risk group required treatment escalation at 3.9 (CI: 2.4-6.3) times the rate of the low risk group. FC values were higher in patients later requiring treatment escalation (Table 2), however this finding was not significant whether analysing $\mathrm{CD}(\mathrm{p}=0.63)$ and $\mathrm{UC}(0.09)$ separately, or in all IBD $(\mathrm{p}=0.14)$.

A simple categorisation for all patients as high or low risk may not be the most useful interpretation of the protein expression panels. Subgroups can be identified at particularly high or low risk of aggressive disease tailored to an appropriate level for the intended action to be taken. Supplementary Figure 2 depicts these data in a graphical format. Each subsection represents the results from labelling a proportion of the population as low (x axis) and high risk (y axis). Within each subsection the top left and bottom right numbers denote the percentage of the identified group requiring escalated treatment in the high and low risk groups respectively. The top right number in each subsection represents the relative risk between groups. As an example, identifying the quartiles of patients at highest and lowest risk selects a subset where $52.8 \%$ and $5.8 \%$ respectively required treatment escalation in the first 18 months of treatment, with a relative risk ratio between groups of 9.1 .

Although analysing all IBD patients (Supplementary Figure S3) in this cohort together produces models which work in both CD and UC, the accuracy achieved in UC is significantly higher than that in CD (85.1\%, CI: 79.2-91.0 vs $70.9 \%$, CI: $62.4-79.4 ; \chi^{2}$ $\mathrm{p}=0.007$ ). The same analytical approach applied individually to UC and CD produces simpler models (2 and 3 proteins respectively, Supplementary Figure S4), with 79.4\% (CI: 72.886.1) accuracy in UC outperforming accuracy in $\mathrm{CD}(76.4 \% \mathrm{CI}: 68.4-84.3)$. As with the panIBD analysis, the probes selected by the inner cross-validation loops were consistent with CD6 and CSF1 in 92\% of UC models and LITAF, CPM, and CCL28 in 99, 97, and 88\% of CD models respectively. 
We compared the performance of PEA based prognostic proteins to currently available blood and faecal biomarkers and clinical predictors in IBD and its subtypes; these are summarised in Supplementary Table 7. The performance of the PEA model is comparable to hsCRP (HR 2.74, CI:1.32-5.67 vs 6-protein model HR 3.90, CI:2.43-6.26). hsCRP however suffers from poor sensitivity (0.20; CI: 11.1-33.1) compared to the PEA model (sensitivity $0.48, \mathrm{CI}$ : 35.3-60.0). A Cox model trained with FC or a combined model with FC and hsCRP performed poorly at predicting treatment escalation in IBD (FC HR 1.17, CI: 0.42-3.26; FC\& hsCRP model HR 0.74, CI:0.18-3.08 respectively). Clinical predictors such as non-B1 behaviour or perianal disease in $\mathrm{CD}$, and SCCAI or HBI scores did not significantly associate with treatment escalation, though pancolitis in UC did (uncorrected $\mathrm{p}=0.002$ ).

Compared to the overall PEA-protein model accuracy of $80.0 \%$, the addition of FC, CRP, or both did not improve model performance yielding accuracies of 76.5\% (CI: 67.5-85.5) 77.8\% (CI: 72.8-82.8), and 72.2\% (CI: 62.3-82.0) respectively, neither did the addition of any phenotypic characteristic such as pancolitis in UC or perianal disease in CD. We also performed correlation analyses of the top protein markers with proteins associated with IBD, hsCRP, albumin, and FC and these are summarised in Supplementary Figure S5.

\section{Circulating proteins associate with germline variation}

It has been shown that expression of proteins associate with germline variation, mainly in the cis regions of their encoding genes ${ }^{22}$. We explored the influence of germline variation on the expression of key IBD diagnostic and prognostic proteins identified in our analysis. We used linear regression models with age and sex as covariates, to analyse SNPs (MAF >0.1) correlated with protein expression, revealing 769 significant cis pQTLs affecting 51 proteins. These included 59 significant cis pQTLs affecting 9 proteins with significant expression changes associated with IBD, (Supplementary Figure S6, Supplementary Table 10), and 
35 pQTLs affecting proteins implicated in disease course (Supplementary Figures S7).

Vascular Endothelial Growth Factor-A (VEGF-A) showed the most significant association with genotype (lead SNP rs7767396; effect $\left.(\beta)-0.42 ; \mathrm{MAF}=0.46 ; \mathrm{p}=8.7 \times 10^{-18}\right)$ with a total of 6 significant SNP associations and 14 SNPs in linkage disequilibrium with rs 7767396.

Among the proteins individually significantly associated with aggressive disease (Table 3 ) or frequently selected in the multi-protein models for aggressive disease significant pQTLs were found in CD6, RANK and SLAMF7 (Supplementary Figure S7), in addition to the findings described in CCL23 above (Supplementary Figure S6).

\section{Discussion}

With advances in clinical care in IBD, it is widely recognised that there is a need for biomarkers that provide accurate diagnostic and prognostic testing in IBD. The key innovation in this study is the design and evaluation of a novel multi-protein panels in newly diagnosed IBD, chosen a priori on the basis of known or suspected involvement in pathogenesis. The results substantiate the involvement of key pathways in pathogenesis, as well as provide targets for therapy. Importantly, we demonstrate that this strategy of biomarker discovery is feasible in diagnosis and in predicting treatment escalation in CD and UC.

A panel of 6 proteins had $79.8 \%$ accuracy, $83.1 \%$ sensitivity, and $74.8 \%$ specificity at differentiating IBD from controls. Whilst FC did outperform this panel (86.4\% accuracy, $85.4 \%$ sensitivity, $88.4 \%$ specificity), uptake was low, overall with patient acceptability a major limiting factor. Given this widely recognised limitations of FC testing in clinic $^{10,11}$, we suggest a serum protein biomarker panel could prove clinically useful as a diagnostic test in replacement of FC. Further studies are now needed to test and validate the utility of this protein panel in clinical practice. 
Of the 66 differentially expressed proteins in IBD, 9 demonstrated germline variation, VEGF-

A being the most significant pQTL. Weaker correlations between protein expression and genetic variation were observed in 4 of the proteins that predicted treatment escalation including CCL23, RANK, CD6 and SLAM7. It is yet to be determined whether these genetic associations are causal in both disease onset and course and our study provide a resource to investigate these associations further.

The greatest unmet need is for biomarkers that can determine disease activity, behaviour and extent, and most critically to predict response to treatment. In our dataset, we have been able to characterise and rigorously cross-validate models involving a limited number of proteins that predict disease course. The role of biomarkers in predicting the disease course has been the focus of many studies ${ }^{2-7,23,24}$, including our own parallel studies of glycomic and methylation profiling in the EC-funded consortia ${ }^{23,25}$. Lee et al identified expression profiles of $\mathrm{T}$ cell exhaustion in $\mathrm{CD} 8 \mathrm{~T}$ cells that predicted treatment escalation in $\mathrm{IBD}^{3}$, defining escalation as the need for 2 or more immunosuppressants and/or surgery after initial disease remission. A multi-gene signature predicting need for escalation using these original criteria has been proposed by this team in UC (HR 3.1, 95\% CI: 1.25-7.72, p=0.02) and CD (HR 2.7; CI: $1.32-5.34, p=0.01)^{7}$. This signature differs from the original profile of T cell exhaustion. Other studies focus on mucosal healing, response to biological agents, and development of fistulising or stricturing complications as end-points - all valid in context.

In this study we decided to use more stringent criteria for escalation than those used in defining the transcriptional profile. We highlight need for biologics or ciclosporin or surgical resection, rather than introduction of immunosuppression per se. This decision regarding endpoint relates principally to the variable threshold for initiating immuno-modulators, which in practice have often been used as first-line therapy in CD. Our oligo-protein panels have the 
potential for clinical translation with significant practical benefits including the simplicity of the assay, and the ability to multiplex proteins using only $1 \mu \mathrm{L}$ of serum.

It is noteworthy that the key prognostic proteins identified relate to pathways independent of TNF signalling. OSM is a pro-inflammatory cytokine that promotes production of IL-6 to attract immune cells to the site of inflammation ${ }^{26}$ and its intestinal expression in IBD has been shown to predict anti-TNF non-response in $\mathrm{IBD}^{26}$. We report that circulating levels of both IL-6 and OSM can predict treatment escalation in IBD. Similarly, we demonstrate the involvement of other pathways that predict disease course (Table 3, Supplementary Figure S8). Of particular relevance are the proteins that show poor correlation with conventional inflammatory markers including hsCRP (Supplementary Figure S5), in particular PSGL-1. This protein is a P-selectin glycoprotein ligand that is expressed on the surface of most immune cells and facilitates immune cell trafficking across the endothelium ${ }^{27,28}$. Drug targeting PSGL-1 is currently in phase 1 trial for the treatment of CD (NIH \#8307272). Future studies examining the performance of these markers in predicting response to therapy are now needed.

We recognise that clinical decisions and timing on treatment escalations may vary across centres. In this study all sites utilised a 'step-up approach' to treatment escalation, rather than a top-down approach. In this respect the clinical management is similar across centres and the consistency of our biomarker profile in predicting need for escalation across centres is especially noteworthy. Our study was not designed to detect the association between prognosis and endoscopic activity. Recently, a protein based endoscopic healing index (EHI) has been reported that incorporates 13 proteins and performs at par with $\mathrm{FC}$ in predicting endoscopic disease remission (validation cohort AUROC, 0.803 for EHI vs AUROC, 0.854 for FC; $\mathrm{P}=0.298$ ); highlighting the translational potential of blood-based protein biomarkers 
in IBD $^{29}$. The predictive capacity of our PEA model performs at par with conventional blood tests such as hsCRP. It is however worth noting that CRP suffers from poor sensitivity $(0.20$; CI: 11.1-33.1) compared to the PEA model (sensitivity 0.48, CI: 35.3-60.0). Other markers such as FC suffer from poor uptake with only $85 \mathrm{FC}$ results available for prognostic analysis in our study. Therefore, a blood-based PEA panel would be better at identifying patients likely to require treatment escalation. As the results of locally analysed CRP and albumin were known to clinicians making treatment decisions regarding escalation of therapy, it is likely these were in fact key determinants in decision-making. Since these measures are often regarded as proxies of inflammatory activity in clinical practice, these markers cannot be considered as independent predictors of disease progression._Our protein markers still remain significant predictors of treatment escalation, independent of clinical confounders. We have utilised nested leave-one-out cross-validation which is acknowledged to produce an unbiased estimate of true error when properly nested so that the entire feature selection and parameter tuning process takes place without reference to the left out samples ${ }^{30}$. This methodology avoids biased estimates of performance and prevents over-fitting of the proposed models. Further validation is now needed to replicate our findings in other large multi-centre inception studies. The significance and impact of our analysis are strengthened by the preestablished evidence for these proteins in IBD or IBD-related pathways. Due to propriety reasons, details of antibodies used for Olink panels were not available, however certain panels including the Olink Inflammation panel are now commercially available for further external validation._ This is however the largest inception cohort recruited in biomarker studies in adult IBD to date, allowing robust modelling and rigorous application.

With advances in IBD therapeutics, future challenges will include tailoring therapies based on individual disease biology. Our data provide an insight into the importance of molecular characterisation of patients with IBD at diagnosis to tailor medical therapies. These data also 
provide substantial progress towards the goal of developing a composite biomarker panel informing patients of their diagnosis and prognosis at their first clinic visit. In $C D$, the PROFILE trial is a landmark prospective biomarker-stratified study in IBD, using the Predict Immune panel, currently recruiting across $\mathrm{UK}^{24}$. Our data provide an impetus for a similar protein-based biomarker trial both in $\mathrm{CD}$ and $\mathrm{UC}$; and provide a rationale for multi-omic profiling to be integrated into trial design, and then into practice. The clear aspiration is that stratification with multi-omic biomarkers based on underlying disease mechanisms may enable personalised therapeutics. 
Author Contributions: Study design RK, JH, MDA, MV, JS. Patient recruitment and sample processing NTV, RK, NAK, DB, SV, ATA. Experimental work OA, FH, CP, RK, NTV, ATA, NAK. Data Analysis RK, NAK, ATA, DR, JL, DB. RK and ATA wrote the manuscript. All authors were involved in critical review, editing, revision and approval of the final manuscript.

Conflict of interest: R. Kalla Financial support for research: EC IBD-Character, Lecture fee(s): Ferring, N. Kennedy Financial support for research: Wellcome Trust, Conflict with: Pharmacosmos, Takeda, Janssen, Dr Falk speaker fees. Abbvie, Janssen travel support, A. Adams: None Declared, J. Satsangi Financial support for research: EC grant IBD-BIOM, Wellcome, CSO, MRC, Conflict with: Consultant for: Takeda, Conflict with: MSD speaker fees. Shire travelling expenses

Funding: The study has been funded by the following EU FP7 grant: IBD-CHARACTER (contract \# 2858546). NAK was funded by the Wellcome Trust (grant number WT097943MA).

Data accessibility statement: Study data can be shared on request to the corresponding author with permission from the IBD Character data access committee. 


\section{Manuscript Doi: 10.1093/ecco-jcc/jjaa230}

Table 1: Demographics and clinical characteristic of patients with newly diagnosed inflammatory bowel disease and symptomatic controls.

\begin{tabular}{|c|c|c|}
\hline & $\begin{array}{l}\text { Inflammatory Bowel Diseases } \\
(\mathrm{n}=328)\end{array}$ & $\begin{array}{l}\text { Symptomatic } \\
\text { Controls } \\
(\mathrm{n}=224)\end{array}$ \\
\hline Mean age (range), years & $34(7-78)$ & $34(3-79)$ \\
\hline Males (\%) & $172(52 \%)$ & $104(46 \%)$ \\
\hline $\begin{array}{l}\text { Smoking status (current: never: ex: } \\
\text { missing) }\end{array}$ & 53:139:107:29 & $48: 100: 56: 20$ \\
\hline $\begin{array}{l}\text { High sensitivity c-reactive protein: } \\
\text { Median (range) }\end{array}$ & $22(0-300)$ & $5(0-85)$ \\
\hline Albumin: Median (range) & $37(13-50)$ & $40(29-52)$ \\
\hline Faecal calprotectin: Median (range) & $1298(32-6001)$ & $78.5(4-2647)$ \\
\hline \multicolumn{3}{|l|}{ Subtype of IBD } \\
\hline Crohn's Disease & $146(45 \%)$ & \\
\hline Ulcerative colitis & $153(47 \%)$ & \\
\hline $\begin{array}{l}\text { Inflammatory Bowel Disease } \\
\text { Unclassified (IBDU) }\end{array}$ & $29(8 \%)$ & \\
\hline Treatment naïve & $235(72 \%)$ & \\
\hline \multicolumn{3}{|l|}{ CD location at Diagnosis } \\
\hline L1 (terminal ileum) & $46(32 \%)$ & \\
\hline L2 (colon) & $43(29 \%)$ & \\
\hline L3 (ileocolon) & $53(36 \%)$ & \\
\hline L4 (Upper GI) & $4(3 \%)$ & \\
\hline \multicolumn{3}{|l|}{ CD Behaviour at Diagnosis } \\
\hline $\begin{array}{l}\text { B1, B1p (non-stricturing \& non- } \\
\text { penetrating, +perianal) }\end{array}$ & $111,6(76 \%, 4 \%)$ & \\
\hline B2, B2p (stricturing, +perianal) & $12,0(8 \%, 0 \%)$ & \\
\hline B3, B3p (penetrating, +perianal) & $7,6(5 \%, 4 \%)$ & \\
\hline Not available & $4(3 \%)$ & \\
\hline \multicolumn{3}{|l|}{ Extent for UC at Diagnosis } \\
\hline E1 (proctitis) & $39(25 \%)$ & \\
\hline
\end{tabular}


Manuscript Doi: 10.1093/ecco-jcc/jjaa230

\begin{tabular}{|l|l|l|}
\hline E2 $($ left sided) & $47(31 \%)$ & \\
\hline E3 (extensive colitis) & $63(41 \%)$ & \\
\hline Not available & $4(3 \%)$ & \\
\hline Centre & & 74 \\
\hline Edinburgh, UK & 107 & 60 \\
\hline Oslo, Norway & 119 & 30 \\
\hline Orebro, Sweden & 57 & 23 \\
\hline Linkoping, Sweden & 16 & 37 \\
\hline Zaragosa, Spain & 24 & 0 \\
\hline Maastricht, Netherlands & 5 & \\
\hline
\end{tabular}

NA: Not applicable; CD: Crohn's disease; UC: Ulcerative colitis; IBDU: Inflammatory bowel disease unclassified. 
Table 2: Demographics and clinical characteristics of patients with inflammatory bowel disease who were included in the predictive analysis of disease course

\begin{tabular}{|l|c|c|}
\hline \multicolumn{2}{|c|}{ Inflammatory Bowel Disease } \\
\hline Males (\%) & $\begin{array}{c}\text { IBD escalation group } \\
(\mathbf{n = 6 7 )}\end{array}$ & $\begin{array}{c}\text { IBD Non-escalation group } \\
(\mathbf{n = 2 1 2})\end{array}$ \\
\hline $\begin{array}{l}\text { Smoking status (current: never: ex: } \\
\text { missing) }\end{array}$ & $39(58)$ & $109(51)$ \\
\hline Median FC (range) & $16: 34: 16: 1$ & $36: 98: 77: 1$ \\
\hline Median age (range) & $1631(35-6001)$ & $1186(32-6001)$ \\
\hline Edin: Norway: Sweden: Spain & $26: 22: 15: 4$ & $33(18-77)$ \\
\hline Disease subtype & & $81: 71: 41: 19$ \\
\hline Crohn's disease & 39 & 73 \\
\hline Ulcerative colitis & 26 & 22 \\
\hline Inflammatory bowel disease \\
unclassified (IBDU)
\end{tabular}

\begin{tabular}{|l|c|c|}
\hline & Uscalation group (n=26) & $\begin{array}{c}\text { Non-escalation group } \\
\text { (n=117) }\end{array}$ \\
\hline Males (\%) & $19(73)$ & $67(57)$ \\
\hline $\begin{array}{l}\text { Smoking status (current: never: ex: } \\
\text { missing) }\end{array}$ & $3: 9: 14: 0$ & $8: 53: 56: 0$ \\
\hline Median FC (range) & $3778(35-6001)$ & $1367(32-6001)$ \\
Median age (range) & $30(18-60)$ & $37(18-77)$ \\
\hline Edin: Norway: Sweden: Spain & $13: 8: 4: 1$ & $39: 52: 19: 7$ \\
\hline Paris Extent for UC & & $38(32 \%)$ \\
\hline E1 (proctitis) & 0 & $37(32 \%)$ \\
\hline E2 (left sided) & $19(73 \%)$ & $42(36 \%)$ \\
\hline E3 (pancolitis) & & \\
\hline
\end{tabular}




\begin{tabular}{|l|c|c|}
\hline & Escalation group (n=39) & Non-escalation group (n=73) \\
\hline Males (\%) & $19(49)$ & $33(45)$ \\
\hline $\begin{array}{l}\text { Smoking status (current: } \\
\text { never: ex: missing) }\end{array}$ & $13: 5: 20: 1$ & $26: 18: 28: 1$ \\
\hline $\begin{array}{l}\text { Median FC (range) } \\
\text { Median age (range) }\end{array}$ & $1398.5(47-6001)$ & $825(70-6001)$ \\
\hline Edin: Norway: Sweden: Spain & $25(18-66)$ & $29(18-73)$ \\
\hline $\begin{array}{l}\text { Montreal classification for CD } \\
\text { L1 (terminal ileum) }\end{array}$ & $11: 14: 11: 3$ & $34: 17: 12: 10$ \\
\hline L2 (colonic) & $13(33 \%)$ & $22(34 \%)$ \\
\hline L3 (ileocolon) & $9(23 \%)$ & $25(34 \%)$ \\
\hline L4 (upper GI) & $17(44 \%)$ & $1(1 \%)$ \\
\hline $\begin{array}{l}\text { Montreal Behaviour for CD } \\
\text { B1, B1p (non-stricturing \& } \\
\text { non-penetrating, +perianal) }\end{array}$ & $29,0(74 \%, 0 \%)$ & $5,6(75 \%, 8 \%)$ \\
\hline B2, B2p (stricturing, +perianal) & $6,0(15 \%, 0 \%)$ & $5,1(7 \%, 1 \%)$ \\
\hline $\begin{array}{l}\text { B3, B3p (penetrating, } \\
+ \text { perianal) }\end{array}$ & $2,2(5 \%, 5 \%)$ & $2(3 \%)$ \\
\hline Not available & 0 & \\
\hline
\end{tabular}




\section{Manuscript Doi: 10.1093/ecco-jcc/jjaa230}

Table 3: Top 15 proteins associated with escalation in treatment (anti-TNF/ciclosporin and/or surgery) and their associated biology based on the available literature. Holm P represents p values adjusted for multiple testing. Log2 HR (hazard ratio) is the relative risk associated with a one unit increase in expression of the relevant protein.

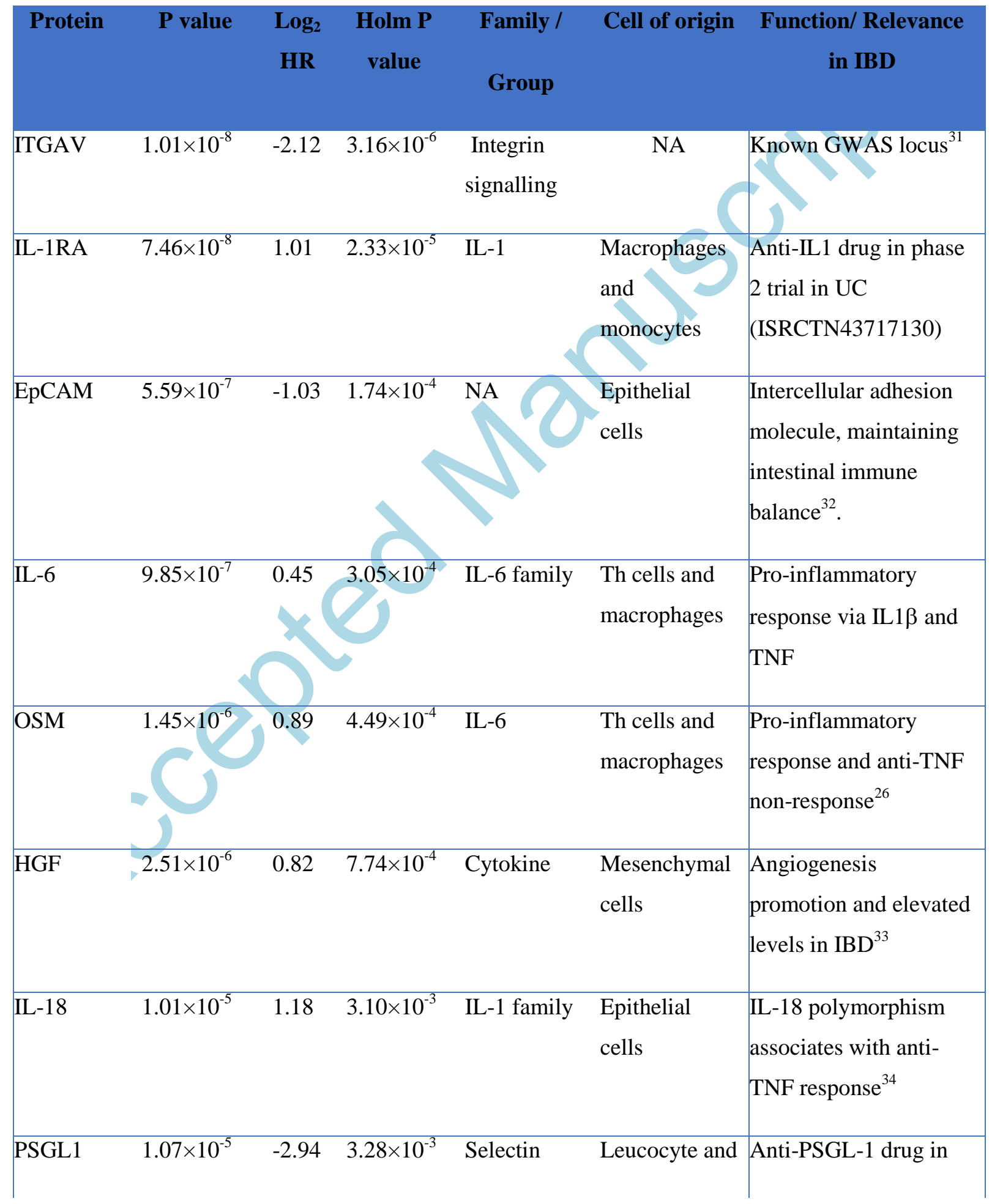


Manuscript Doi: 10.1093/ecco-jcc/jjaa230

\begin{tabular}{|c|c|c|c|c|c|c|}
\hline & & & & family & $\begin{array}{l}\text { endothelial } \\
\text { surfaces }\end{array}$ & $\begin{array}{l}\text { Phase } 1 \text { trial to treat } \\
\mathrm{CD}(\mathrm{NIH} \# 8307272)\end{array}$ \\
\hline ADM & $1.16 \times 10^{-5}$ & 1.02 & $3.53 \times 10^{-3}$ & $\begin{array}{l}\text { Calcitonin } \\
\text { peptide } \\
\text { superfamily }\end{array}$ & $\begin{array}{l}\text { Epithelial } \\
\text { cells }\end{array}$ & $\begin{array}{l}\text { Case series of mucosal } \\
\text { healing in refractory } \\
\text { UC with AM therapy }\end{array}$ \\
\hline CSF-1 & $1.20 \times 10^{-5}$ & 1.04 & $3.64 \times 10^{-3}$ & $\begin{array}{l}\text { IL-34/CSF- } \\
1 \text { family }\end{array}$ & $\begin{array}{l}\text { Various } \\
\text { immune cells }\end{array}$ & $\begin{array}{l}\text { Pro-inflammatory } \\
\text { macrophage induced } \\
\text { response }^{36}\end{array}$ \\
\hline TNF-R1 & $1.89 \times 10^{-5}$ & 1.21 & $5.74 \times 10^{-3}$ & TNF family & $\begin{array}{l}\text { Macrophages } \\
\text { and dendritic } \\
\text { cells }\end{array}$ & $\begin{array}{l}\text { Pro-inflammatory TNF } \\
\text { mediated response }\end{array}$ \\
\hline CCL23 & $5.38 \times 10^{-5}$ & 0.81 & 0.016 & $\begin{array}{l}\mathrm{CC} \\
\text { chemokin }\end{array}$ & $\begin{array}{l}\text { Epithelial and } \\
\text { immune cells }\end{array}$ & $\begin{array}{l}\text { Neutrophil activation } \\
\text { and leukocyte } \\
\text { migration }^{37}\end{array}$ \\
\hline IL-8 & $6.98 \times 10^{-5}$ & 0.52 & 0.021 & & $\begin{array}{l}\text { Epithelial } \\
\text { cells, } \\
\text { macrophages, } \\
\text { monocytes }\end{array}$ & $\begin{array}{l}\text { Neutrophil recruitment } \\
\text { and pro-inflammatory } \\
\text { response }\end{array}$ \\
\hline$\overline{\mathrm{CPM}}$ & $7.64 \times 10^{-5}$ & & 0.023 & $\begin{array}{l}\text { Carboxy } \\
\text { peptidases }\end{array}$ & $\begin{array}{l}\text { Activated } \\
\text { macrophages }\end{array}$ & $\begin{array}{l}\text { Activated macrophage } \\
\text { differentiating marker }\end{array}$ \\
\hline IL-17D & $1.22 \times 10^{-4}$ & -2.25 & 0.036 & $\begin{array}{l}\text { IL-17 } \\
\text { family }\end{array}$ & Th-17 cells & $\begin{array}{l}\text { Th-17 driven pro- } \\
\text { inflammatory cytokine }\end{array}$ \\
\hline
\end{tabular}




\section{Manuscript Doi: 10.1093/ecco-jcc/jjaa230}

\section{Figure Legends}

Figure 1: A) Volcano plot displaying the $\log _{2}$ fold-change and significance of protein associations with IBD.

Dotted line indicates threshold for significance (Holm p<0.05) after Holm correction derived by

$\log 10\left(\right.$ uncorrected $\left.\mathrm{p}=1.78 \times 10^{-4}\right)$. B) Fold change between Ulcerative colitis(UC) and Crohn's disease(CD)

respectively vs controls, points coloured by significance after Holm correction in CD, UC, both, or neither (ns).

Figure 2: The significance of protein markers in predicting treatment escalation in inflammatory bowel disease and ulcerative colitis. Significance threshold after Holm correction indicated by dotted line. 


\section{References}

1. Boyapati RK., Kalla R., Satsangi J., Ho G-T. Biomarkers in Search of Precision Medicine in IBD. Am J Gastroenterol 2016;111(12):1682-90. Doi: 10.1038/ajg.2016.441.

2. Kalla R., Kennedy NA., Ventham NT., Boyapati RK., Adams AT., Nimmo ER., et al. Serum Calprotectin: A Novel Diagnostic and Prognostic Marker in Inflammatory Bowel Diseases. Am J Gastroenterol 2016;111(12):1796-805. Doi: 10.1038/ajg.2016.342.

3. Lee JC., Lyons P a., McKinney EF., Sowerby JM., Carr EJ., Bredin F., et al. Gene expression profiling of CD8+T cells predicts prognosis in patients with Crohn disease and ulcerative colitis. J Clin Invest 2011;121(10):4170-9. Doi: 10.1172/JCI59255.

4. Lee JC., Biasci D., Roberts R., Gearry RB., Mansfield JC., Ahmad T., et al. Genome-wide association study identifies distinct genetic contributions to prognosis and susceptibility in Crohn's disease. Nat Genet 2017;49(2):262-8. Doi: 10.1038/ng.3755.

5. Kugathasan S., Denson LA., Walters TD., Kim M-O., Marigorta UM., Schirmer M., et al. Prediction of complicated disease course for children newly diagnosed with Crohn's disease: a multicentre inception cohort study. Lancet (London, England) 2017;389(10080):1710-8. Doi: 10.1016/S0140-6736(17)30317-3.

6. Marigorta UM., Denson LA., Hyams JS., Mondal K., Prince J., Walters TD., et al. Transcriptional risk scores link GWAS to eQTLs and predict complications in Crohn's disease. Nat Genet 2017;49(10):1517-21. Doi: 10.1038/ng.3936.

7. Biasci D., Lee JC., Noor NM., Pombal DR., Hou M., Lewis N., et al. A blood-based prognostic biomarker in IBD. Gut 2019;68(8):1386-95. Doi: 10.1136/gutjnl-2019-318343.

8. Colombel J-F., Panaccione R., Bossuyt P., Lukas M., Baert F., Vaňásek T., et al. Effect of tight control management on Crohn's disease (CALM): a multicentre, randomised, controlled phase 3 trial. Lancet 2017;390(10114):2779-89. Doi: 10.1016/S0140-6736(17)32641-7. 
9. van Rheenen PF., Van de Vijver E., Fidler V. Faecal calprotectin for screening of patients with suspected inflammatory bowel disease: diagnostic meta-analysis. BMJ 2010;341:c3369.

10. Kalla R., Boyapati R., Vatn S., Hijos G., Crooks B., Moore GT., et al. Patients' perceptions of faecal calprotectin testing in inflammatory bowel disease: results from a prospective multicentre patient-based survey. Scand J Gastroenterol 2018:1-6. Doi: 10.1080/00365521.2018.1527394.

11. Maréchal C., Aimone-Gastin I., Baumann C., Dirrenberger B., Guéant J-L., Peyrin-Biroulet L. Compliance with the faecal calprotectin test in patients with inflammatory bowel disease. United Eur Gastroenterol J 2017:205064061668651. Doi: 10.1177/2050640616686517.

12. Assarsson E., Lundberg M., Holmquist G., Björkesten J., Thorsen SB., Ekman D., et al. Homogenous 96-plex PEA immunoassay exhibiting high sensitivity, specificity, and excellent scalability. PLoS One 2014;9(4):e95192. Doi: 10.1371/journal.pone.0095192.

13. Lundberg M., Eriksson A., Tran B., Assarsson E., Fredriksson S. Homogeneous antibody-based proximity extension assays provide sensitive and specific detection of low-abundant proteins in human blood. Nucleic Acids Res 2011;39(15):e102. Doi: 10.1093/nar/gkr424.

14. Satsangi J., Silverberg MS., Vermeire S., Colombel J-F. The Montreal classification of inflammatory bowel disease: controversies, consensus, and implications. Gut 2006;55(6):749-53. Doi: 10.1136/gut.2005.082909.

15. Levine A., Griffiths A., Markowitz J., Wilson DC., Turner D., Russell RK., et al. Pediatric modification of the Montreal classification for inflammatory bowel disease: the Paris classification. Inflamm Bowel Dis 2011;17(6):1314-21. Doi: 10.1002/ibd.21493.

16. Maaser C., Sturm A., Vavricka SR., Kucharzik T., Fiorino G., Annese V., et al. ECCO-ESGAR Guideline for Diagnostic Assessment in IBD Part 1: Initial diagnosis, monitoring of known IBD, detection of complications. J Crohns Colitis 2019;13(2):144-64. Doi: 10.1093/ecco-jcc/jjy113. 
17. Jostins L., Ripke S., Weersma RK., Duerr RH., McGovern DP., Hui KY., et al. Host-microbe interactions have shaped the genetic architecture of inflammatory bowel disease. Nature 2012;491(7422):119-24. Doi: 10.1038/nature11582.

18. Thorsen SB., Lundberg M., Villablanca A., Christensen SLT., Belling KC., Nielsen BS., et al. Detection of serological biomarkers by proximity extension assay for detection of colorectal neoplasias in symptomatic individuals. J Transl Med 2013;11:253. Doi: 10.1186/1479-587611-253.

19. Bezanson J., Edelman A., Karpinski S., Shah VB. Julia: A Fresh Approach to Numerical Computing. SIAM Rev 2017;59(1):65-98. Doi: 10.1137/141000671.

20. Holm S. A simple sequentially rejective multiple test procedure. Scand J Stat 1979;6(2):65-70.

21. Shabalin AA. Matrix eQTL: ultra fast eQTL analysis via large matrix operations. Bioinformatics 2012;28(10):1353-8. Doi: 10.1093/bioinformatics/bts163.

22. Sun BB., Maranville JC., Peters JE., Stacey D., Staley JR., Blackshaw J., et al. Genomic atlas of the human plasma proteome. Nature 2018;558(7708):73-9. Doi: 10.1038/s41586-018-01752.

23. Clerc F., Novokmet M., Dotz V., Reiding KR., de Haan N., Kammeijer GSM., et al. Plasma NGlycan Signatures Are Associated With Features of Inflammatory Bowel Diseases. Gastroenterology 2018;155(3):829-43. Doi: 10.1053/j.gastro.2018.05.030.

24. Parkes M., Noor NM., Dowling F., Leung H., Bond S., Whitehead L., et al. PRedicting Outcomes For Crohn's dlsease using a moLecular biomarkEr (PROFILE): protocol for a multicentre, randomised, biomarker-stratified trial. BMJ Open 2018;8(12):e026767. Doi: 10.1136/bmjopen-2018-026767.

25. Ventham NT., Kennedy NA., Adams AT., Kalla R., Heath S., O'Leary KR., et al. Integrative epigenome-wide analysis demonstrates that DNA methylation may mediate genetic risk in 
26. West NR., Hegazy AN., Owens BMJ., Bullers SJ., Linggi B., Buonocore S., et al. Oncostatin M drives intestinal inflammation and predicts response to tumor necrosis factor-neutralizing therapy in patients with inflammatory bowel disease. Nat Med 2017;23(5):579-89. Doi: 10.1038/nm.4307.

27. Guyer DA., Moore KL., Lynam EB., Schammel CM., Rogelj S., McEver RP., et al. P-selectin glycoprotein ligand-1 (PSGL-1) is a ligand for L-selectin in neutrophil aggregation. Blood 1996;88(7):2415-21.

28. Brown JB., Cheresh P., Zhang Z., Ryu H., Managlia E., Barrett TA. P-selectin glycoprotein ligand-1 is needed for sequential recruitment of T-helper 1 (Th1) and local generation of Th17 T cells in dextran sodium sulfate (DSS) colitis. Inflamm Bowel Dis 2012;18(2):323-32. Doi: 10.1002/ibd.21779.

29. D'Haens G., Kelly O., Battat R., Silverberg MS., Laharie D., Louis E., et al. Development and Validation of a Test to Monitor Endoscopic Activity in Patients With Crohn's Disease Based on Serum Levels of Proteins. Gastroenterology 2020;158(3):515-526.e10. Doi:

\subsection{3/j.gastro.2019.10.034.}

30. Lachenbruch PA., Mickey MR. Estimation of Error Rates in Discriminant Analysis.

Technometrics 1968;10(1):1-11. Doi: 10.1080/00401706.1968.10490530.

31. de Lange KM., Moutsianas L., Lee JC., Lamb CA., Luo Y., Kennedy NA., et al. Genome-wide association study implicates immune activation of multiple integrin genes in inflammatory bowel disease. Nat Genet 2017;49(2):256-61. Doi: 10.1038/ng.3760.

32. Jiang L., Shen Y., Guo D., Yang D., Liu J., Fei X., et al. EpCAM-dependent extracellular vesicles from intestinal epithelial cells maintain intestinal tract immune balance. Nat Commun 2016;7:13045. Doi: 10.1038/ncomms13045. 
Manuscript Doi: 10.1093/ecco-jcc/jjaa230

33. Srivastava M., Zurakowski D., Cheifetz P., Leichtner A., Bousvaros A. Elevated serum hepatocyte growth factor in children and young adults with inflammatory bowel disease. $J$ Pediatr Gastroenterol Nutr 2001;33(5):548-53.

34. Bank S., Julsgaard M., Abed OK., Burisch J., Broder Brodersen J., Pedersen NK., et al. Polymorphisms in the NFkB, TNF-alpha, IL-1beta, and IL-18 pathways are associated with response to anti-TNF therapy in Danish patients with inflammatory bowel disease. Aliment Pharmacol Ther 2019;49(7):890-903. Doi: 10.1111/apt.15187.

35. Ashizuka S., Inatsu H., Kita T., Kitamura K. Adrenomedullin Therapy in Patients with Refractory Ulcerative Colitis: A Case Series. Dig Dis Sci 2016;61(3):872-80. Doi: 10.1007/s10620-015-3917-0.

36. Marshall D., Cameron J., Lightwood D., Lawson ADG. Blockade of colony stimulating factor-1 (CSF-I) leads to inhibition of DSS-induced colitis. Inflamm Bowel Dis 2007;13(2):219-24. Doi: 10.1002/ibd.20055.

37. Singh UP., Singh NP., Murphy EA., Price RL., Fayad R., Nagarkatti M., et al. Chemokine and cytokine levels in inflammatory bowel disease patients. Cytokine 2016;77:44-9. Doi: 10.1016/j.cyto.2015.10.008.

38. Tsakiris I., Torocsik D., Gyongyosi A., Dozsa A., Szatmari I., Szanto A., et al. Carboxypeptidase$M$ is regulated by lipids and CSFs in macrophages and dendritic cells and expressed selectively in tissue granulomas and foam cells. Lab Invest 2012;92(3):345-61. Doi:

10.1038/labinvest.2011.168.

39. FANTOM Consortium and the RIKEN PMI and CLST (DGT)., Forrest ARR., Kawaji H., Rehli M., Baillie JK., de Hoon MJL., et al. A promoter-level mammalian expression atlas. Nature 2014;507(7493):462-70. Doi: 10.1038/nature13182. 
Figure 1: Proximity Extension Assay (PEA) Diagnostic Marker Analyses
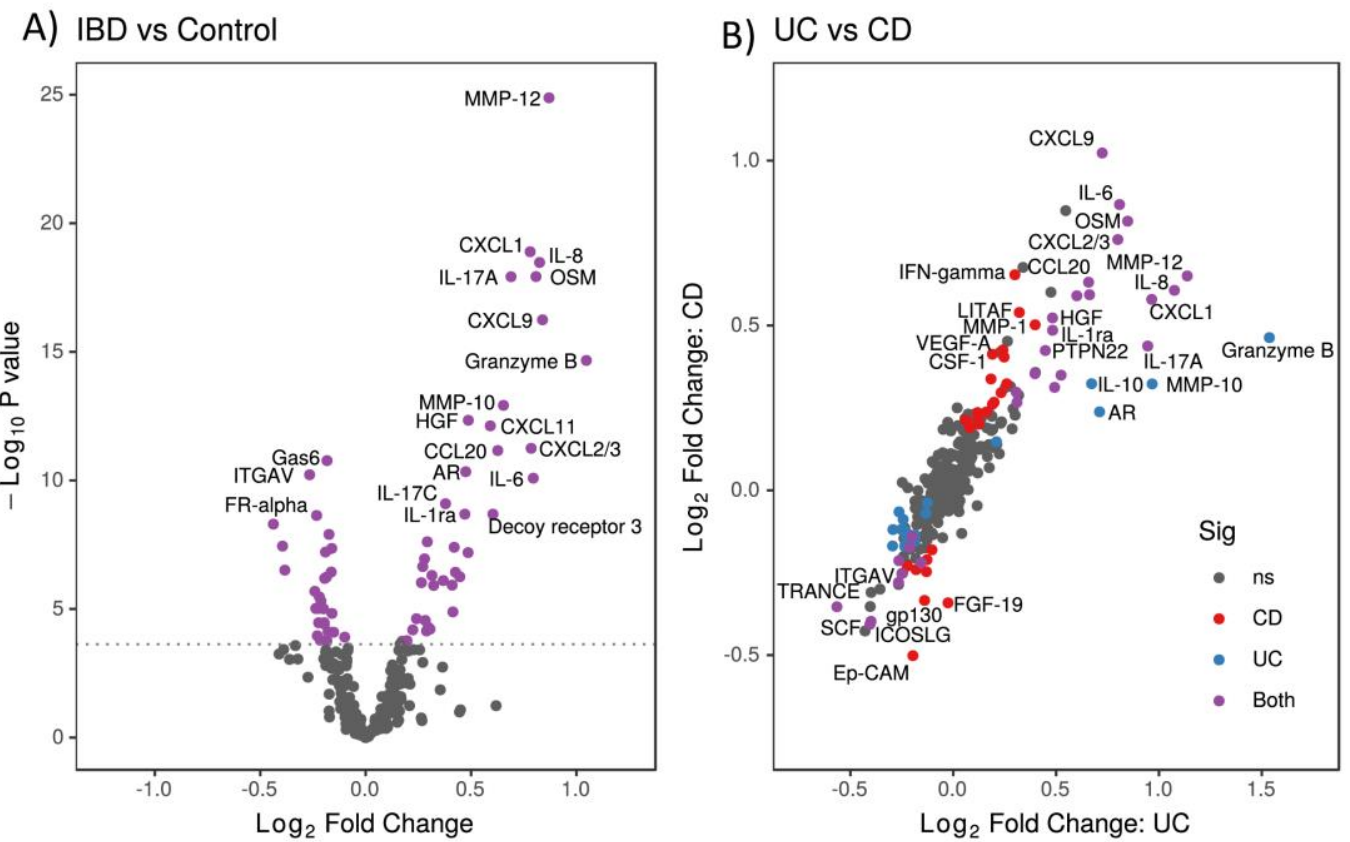
Figure 1: Proximity Extension Assay (PEA) Prognostic Marker Analyses

Inflammatory Bowel Disease

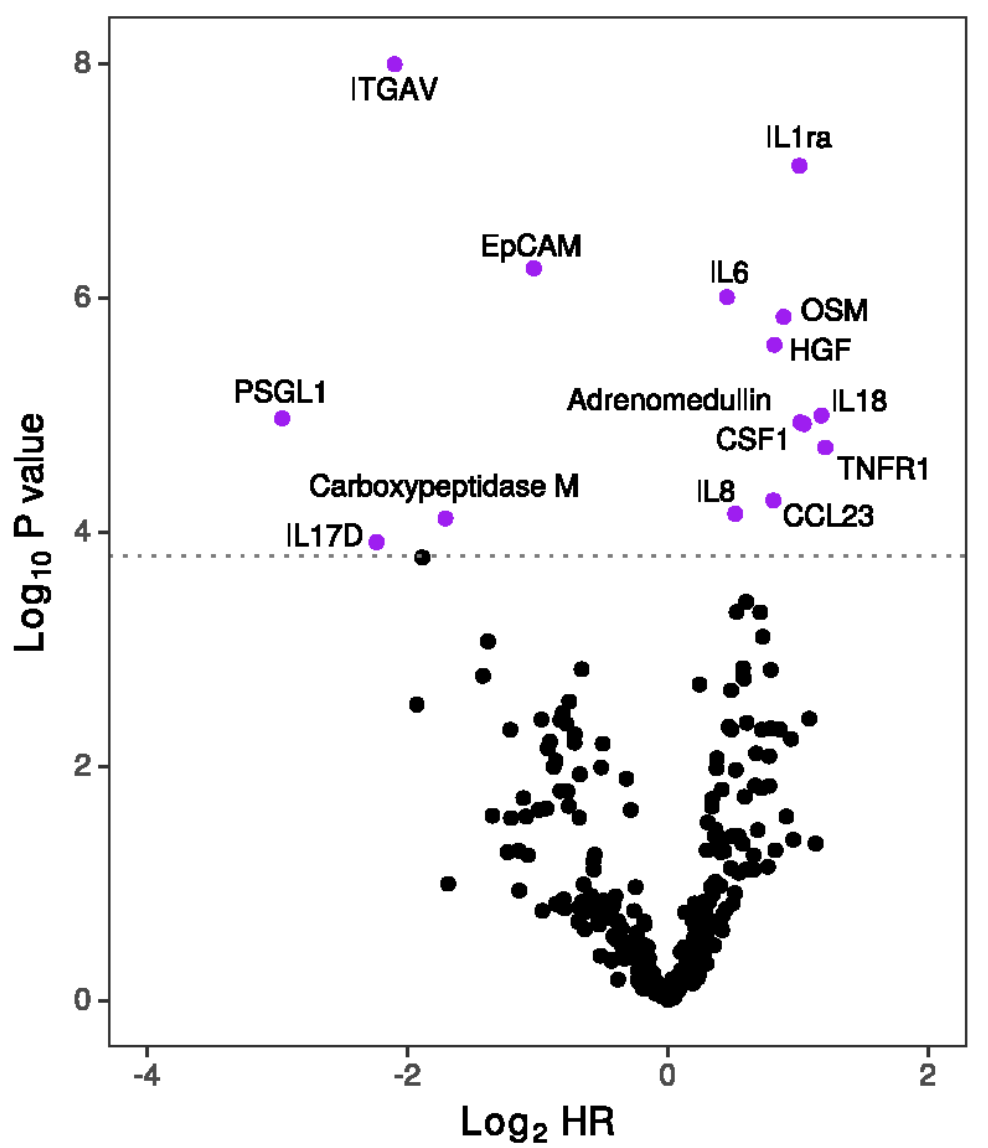

Ulcerative Colitis

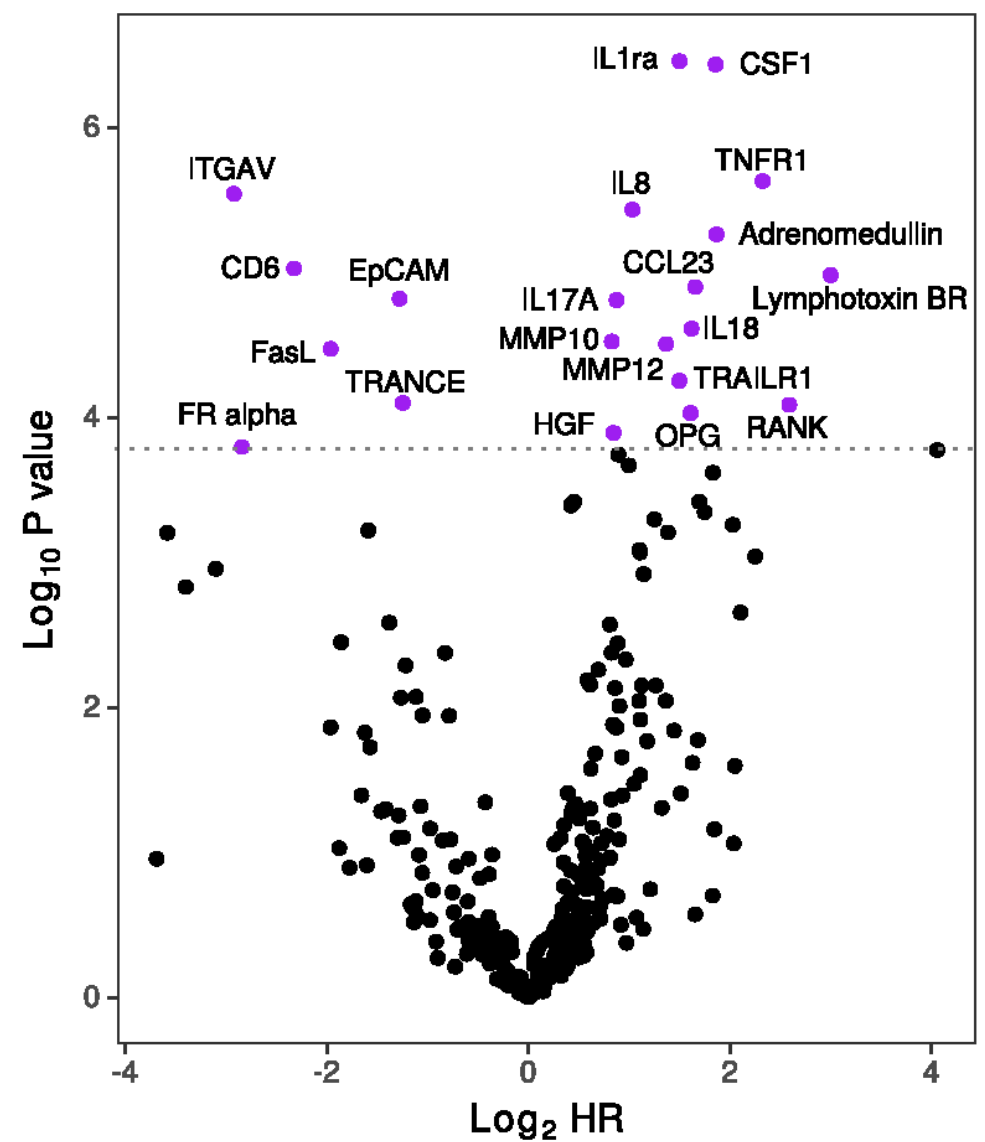

